\title{
"No Innovation Without Cooperation" - How Switzerland Innovation Promotes Cooperation Between Industry, Research and Startups
}

\author{
Raphaël Tschanz ${ }^{\star}$, Stéphanie Cristo, Leonildo Delgado, Vincent Hiroz, Martin Jordan, René Kalt, \\ Isabelle Mitchell, Nina Müller, Claudia Roeoesli, Valentina Tamburello, and Svantje Tauber
}

\begin{abstract}
Switzerland Innovation, the Swiss innovation park with its five branches, is facilitating collaborations for companies, startups, and universities to find solutions to some of the world's most pressing challenges in the fields of health and the life sciences, in particular in the areas of chemistry, biochemistry, biomedicine, biotech, medtech and digital health. Together with its numerous and diverse partners, Switzerland Innovation creates an ecosystem for universities and research-based companies, accelerating the transformation of research results into marketable products and services.
\end{abstract}

Keywords: Accelerator $\cdot$ Incubator $\cdot$ Innovation park $\cdot$ Startups $\cdot$ Ventures

\section{Switzerland Innovation - The Swiss Innovation Park}

Switzerland Innovation forms a network of ecosystems that merges talent, knowledge and expertise in order to create new technology-driven solutions. The collaboration between the Swiss public and private sectors ensures a winning combination, with the Innovation Parks serving as melting pots for these protagonists. ${ }^{[1]}$ The mission of the organizations working under the umbrella of Switzerland Innovation is to lead the way in terms of technical infrastructure enabling innovation in the field of health and life sciences as one of five focus areas. The network of ecosystems created by Switzerland Innovation fosters collaboration with academic partners and industry, which allows universities and innovative companies to use their competencies for the development of marketable products and services. There are five Switzerland Innovation Parks nationwide, each with its human and technical infrastructure. In this article, we present examples of ventures in the areas of chemistry, biochemistry, and biomedicine emerging from the ecosystems provided by the network of Switzerland Innovation.

Although Venture Kick is not part of the network of Switzerland Innovation, it supports young companies on their journey from science to market with early stage funding. Some of the young companies and spin-offs supported by Venture Kick are based in one of the five Switzerland Innovation Parks.

\section{BaseLaunch - Accelerating Therapeutic Ventures}

At the Switzerland Innovation Park Basel Area innovation and cooperation go hand in hand. With its infrastructure offerings and tailored support for startups, as well as the presence of the biotech incubator and accelerator BaseLaunch, the Switzerland Innovation Park Basel Area helps accelerate the pace of life sciences innovation.

The Switzerland Innovation Park Basel Area and BaseLaunch bring together and cooperate with:[2]

- Entrepreneurs and scientific founders across different experience levels and geographic areas to advance technologies that shape the future of patient care
- Industry professionals, consultants, and service providers with experience across different health-care sectors to help advance and accelerate program development

- Global biopharma companies in areas that are of strategic interest; BaseLaunch deploys seed financing in the incubation or creation of new ventures

- Investment professionals with the goal of helping to form high-valued companies

- Academic research institutions to guide the translation of their research into commercial products and services

- Innovation centers across Europe for access to early-stage scientific breakthroughs

As one of the leading life sciences innovation hubs, the Basel Area is home to over 700 life sciences companies and more than 1,000 research groups. Together with a variety of venture capital firms, it is a breeding ground for the creation and advancement of new companies, ideas, and scientific discoveries in biotech, medtech, digital health, healthtech and industrial transformation. ${ }^{[3]}$

The Innovation Park has four sites within the Basel Area (Allschwil, Basel, Novartis Campus and Jura) in order to leverage the potential of the region. The main site in Allschwil is in the up-and-coming BaseLink area, and will move to a new campus in 2022, built by renowned architects Herzog \& de Meuron. Recent success stories and examples of cooperation between industry, research and startups include a CHF 12.5 million financing round for Alentis Therapeutics AG, CHF 22.5 million for Polyneuron Pharmaceuticals AG, CHF 35 million for Anaveon AG and CHF 12 million for T3 Pharmaceuticals AG. All these companies received early-stage support from BaseLaunch.

\section{Switzerland Innovation Park Innovaare - Drug Development with PSI}

Switzerland Innovation Park Innovaare is located next to the Paul Scherrer Institute (PSI) in Villigen, at the shore of the river Aare. The PSI is the largest research institute for natural and en- 
gineering sciences in Switzerland and conducts research in the fields of matter and materials, energy and the environment, and people and health. The member companies of the innovation park work closely with the PSI as their main academic partner.

\section{- Accelerator Technologies Form the Core of PSI's Research} Facilities

With the Synchrotron Light Source Switzerland SLS, the $\mathrm{X}$-ray free-electron laser SwissFEL, the neutron source SINQ and the muon source S $\mu$ S, PSI operates large-scale scientific research facilities that offer insights into the processes taking place inside various substances and materials. By utilizing PSI's large research facilities and specially developed methods, researchers can observe phenomena that have been unobservable until now. For instance, with the new free electron laser SwissFEL, scientists can see processes as fast as the vibrations of molecular bonds.

- Early-Stage Drug Development at Park Innovaare Switzerland Innovation Park Innovaare has been developing a focused innovation ecosystem providing access to all premises for state-of-the-art pharmacological research. In the field of biochemistry, the companies located in the innovation park are active mainly in early-stage drug discovery. ${ }^{[4]}$

- Structure-based drug discovery on membrane protein targets With the focus on membrane protein drug targets, «leadXpro AG» enables structure-based discovery of novel medicines. Investigation of the interaction of drug candidates with their human disease-relevant proteins using X-ray crystallography, cryo-EM and other biophysical methods fuels computational methods to design drug molecules with unprecedented potency and lower side effects. Recently, leadXpro has successfully identified novel A2B receptor modulators, offering new treatment options against cancer.

\section{- Systems Biology for drug discovery}

PSI spin-off InterAx Biotech is reinventing GPCR drug discovery using mathematical models describing biological processes. For their systems biology platform PICARD, InterAx Biotech received funding from the European Union's Horizon 2020 research and innovation program. With its approach, InterAx Biotech combines systems biology with experimental data and uses data-based methods to design novel compounds with the desired properties.

- Reducing Time and Costs for the Drug Discovery Process With their technology platform, InterAx Biotech offers solutions to pharmaceutical and biotech companies that outsource key stages of their drug discovery process. InterAx Biotech benefits from being closely embedded in the PSI/accelerator environment with experts on structural GPCR biology, both at leadXpro and in the PSI Bio Department. ELDICO Scientific AG is developing an electron diffractometer that will allow investigations on hitherto unmeasurable nanocrystalline systems where conventional methods (X-ray) fail, therefore reducing the time and costs of the drug discovery process.

\section{Switzerland Innovation Park Zurich - Life Science at Zero Gravity}

The Switzerland Innovation Park Zurich (IPZ) is building a platform for science, development, and innovation on the area of the Dübendorf airfield. The IPZ promotes the collaboration and sharing of knowledge between the Zurich universities, science institutions and universities of applied sciences (Fachhochschulen) and private industries. The geographic location with its proximity to the Dübendorf airfield makes the park highly attractive. Accordingly, aerospace is one of the focus topics of the IPZ. In this report, we focus on the activities of the UZH Space Hub, the innovation cluster 'Space and Aviation' of the University of Zurich (UZH), a close partner of the IPZ. Its mission is to bring together students, researchers, companies, and startups, linking the UZH community to industries, to share knowledge and bring innovative research and applications to market.

The infrastructure of the Dübendorf airfield is extensively used by UZH Space Hub and its partners for research and development. The airfield is used for research flights such as the Swiss Parabolic Flights (SPF), conducted with the Airbus A310 Zero-G from Novespace (France). The parabolic maneuver provides reduced gravity, offering a cost-effective possibility to conduct zero-g experiments.

So far, 31 experiments addressing physical, chemical, biological, and medical questions have been conducted by Swiss universities and companies. Among these, the medical experiment of Dr. Jaap Swanenburg and his team from Balgrist University Hospital deserves special attention. The aim of the study was to determine the response of the lumbar spinal motor control under micro-and hypergravity conditions. This was achieved by measuring indicators of lumbar motor control, specifically lumbar spinal stiffness, activity of lumbar muscles and lumbar curvature. ${ }^{[5]}$ Thanks also to the results achieved using the SPF, Dr. Swanenburg was selected to conduct further experiments on a Partial Gravity Parabolic Flight Campaign of the ESA and to conduct pre- and post-flight experiments with astronauts on long-duration International Space Station (ISS) missions. These experiments aim to help understanding the stabilization mechanisms of the spine, which might be useful for the development of rehabilitation programs for patients with low back pain or even 'readaptation to Earth'-programs for astronauts.

Another important experiment, resulting from a public-private partnership, was successfully conducted in spring 2020: Researchers from the UZH Space Hub together with Airbus Defense and Space sent adult human stem cells to the ISS to test the production of human tissue in weightlessness. ${ }^{[6]}$ On Earth, tissues are difficult and costly to produce, as earth's gravity prevents the cells from forming spontaneously into tissues. Although the detailed analysis of the samples is still ongoing, the experiment seems promising, as the cells have indeed formed tissue-like structures. The vision is to produce tissues in space on a large scale on a commercial basis to then apply them in transplantation and precision medicine.

\section{Energypolis Campus - Innovation at the Heart of the Alps}

The Energypolis campus, a member of the Switzerland Innovation Park Network West EPFL, is an innovation ecosystem bringing together the skills of EPFL Valais Wallis, HES-SO Valais-Wallis, The Ark Foundation and many other organizations in the fields of energy, green chemistry and the alpine and polar environment as well as health and rehabilitation. ${ }^{[7]}$

To facilitate the emergence of more efficient models, EPFL Valais Wallis and HES-SO Valais-Wallis regularly launch what are known as demonstrators - full-scale test facilities for real-world testing of the technologies developed within the Energypolis campus laboratories. A number of demonstrators are already operational, such as the 'GridLab', which studies the integration of renewable energies and storage for tomorrow's electricity networks, and the 'Grid2Mobility' demonstrator in Martigny, which aims to offer a complete hydrogen production, storage and distribution chain in a single site.

To further accelerate the technology transfer, the campus will soon include a regional branch of the Switzerland Innovation Park Network West EPFL, with the aim of offering workspaces, scientific infrastructure, and equipment. The Innovation Park in Sion will also host startups and spin-offs from the Energypolis campus as well as R\&D and innovation units from industrial companies.

The Energypolis campus and its partners promote the development of tangible and concrete solutions. As a result, several startups have been successfully launched in Sion, where they are able to benefit from the campus's strong innovation ecosystem. Among them, two very promising spin-off companies from EPFL Valais 
Wallis: SENSàSION, which is developing a new rapid Point-ofCare (POC) device for bacteria detection based on very sensitive electrochemical detection of viable bacteria, and DePoly, which has developed a chemical-based method for recycling PET containers through depolymerization.

\section{Venture Kick - Closing the Gap in the Swiss Innovation Chain}

Venture Kick is a philanthropic initiative that was launched in 2007 with the vision of doubling the number of spin-offs at Swiss universities, accelerating the time to market, and increasing the attractiveness of these young companies to professional investors. In 2020, Venture Kick will pay out CHF 5 million to idea-stage startup projects to bring Swiss science to global markets. ${ }^{[8]}$

With its nine-month kick-start process, Venture Kick supports young entrepreneurs with high-potential business ideas on their journey from science to market. The Venture Kick Foundation identifies, structures and promotes promising businesses with an approach that focuses on business development and includes a three-stage pitch process where Swiss startups can win up to $\mathrm{CHF}$ 150,000 in seed funding, get direct feedback and gain access to an international network of successful entrepreneurs and investors.

The Venture Kick Foundation is supported by 11 private partners, foundations, private individuals and corporations that all share the same vision of supporting the next generation of successful entrepreneurs for the prosperity of Switzerland's economy and society: Gebert Rüf Stiftung, Ernst Göhner Stiftung, HauserStiftung, André Hoffmann, Hansjörg Wyss, Martin Haefner, Igor Fisch, Fondation Pro Techno, ESA BIC Switzerland, Engagement Migros and Swisscom.

Since 2007, Venture Kick has supported more than 700 Swiss startup projects with CHF 30 million of funds. Over $30 \%$ of the supported startups come from the life sciences sector, and they have received more than CHF 12 million of funds. The program's combination of financial support, training and networking has led to the creation of 519 companies and 7,000 jobs. Venture Kick alumni companies have attracted a total of CHF 3.5 billion of funds. In 2019, more than half of the TOP 100 Swiss Startups were Venture Kick winners.

In 2020, Venture Kick introduced Venture Kick Life Science for biotech and medtech startups. This new track strengthens Switzerland's position as Europe's life sciences hub and leverages the support, network, and visibility for life sciences projects.

Received: June 24, 2020

[1] Switzerland Innovation, https://www.switzerland-innovation.com/, accessed Aug. 25, 2020.

[2] BaseLaunch, https://www.baselaunch.ch/, accessed Aug. 25, 2020.

[3] Basel Area, https://innovate.baselarea.swiss/, accessed Aug. 25, 2020.

[4] Switzerland Innovation Park Innovaare, 'Shaping the Future Of Drug Discovery', https://www.parkinnovaare.ch/drug-discovery/, accessed Aug. $25,2020$.

[5] J. Swanenburg, A. Langenfeld, Ch. A. Easthope, M. L. Meier, O. Ullrich, P. Schweinhardt, Front. Physiol. 2020, doi: 10.3389/fphys.2020.562557, https://www.frontiersin.org/articles/10.3389/fphys.2020.562557/abstract, accessed Aug. 25, 2020.

[6] University of Zurich, 'Producing Human Tissue in Space', https://www. media.uzh.ch/en/Press-Releases/2020/UZH-Space-Hub.html, accessed Aug. $25,2020$.

[7] Energypolis, https://www.energypolis.ch/, accessed Aug. 25, 2020.

[8] Venture Kick, https://www.venturekick.ch/, accessed Aug. 25, 2020.

\section{License and Terms}

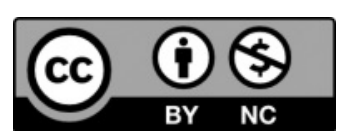

This is an Open Access article under the terms of the Creative Commons Attribution License CC BY_NC 4.0. The material may not be used for commercial purposes.

The license is subject to the CHIMIA terms and conditions: (http:// chimia.ch/component/sppagebuilder/?view=page\&id=12).
The definitive version of this article is the electronic one that can be found at https://doi.org/10.2533/chimia.2020.755

\section{Switzerland Innovation}

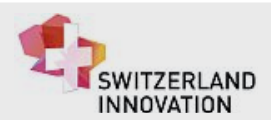

Switzerland Innovation forms an ecosystem that allows universities and innovative companies to collaborate and use their research results for the development of new marketable products and services, provides breeding grounds for innovations and strengthens Switzerland as an innovation country.

\section{Key Figures}

Year of foundation 2015

Location(s)

Headquarters: Bern

Sites: Allschwil, Biel, Lausanne, Villigen, Zurich

\begin{tabular}{l|l|}
\hline Legal Form & Foundation \\
\hline $\begin{array}{l}\text { President } \\
\text { CEO }\end{array}$ & Ruedi Noser \\
\hline
\end{tabular}

\section{Contact}

Contact Person

Address

Raphaël Tschanz, Deputy Head

Switzerland Innovation

Monbijoustrasse 15

3011 Bern

E-mail

info@switzerland-innovation.com

Website www.switzerland-innovation.com

\section{Venture Kick}

\section{VENTURE:०}

Venture Kick aims to promote entrepreneurship at Swiss universities and encourage the creation of highly innovative, science-based startups. Venture Kick helps excellent discoveries and research get funded at the crucial, early stage of their development.

\section{Key Figures}

Year of foundation

2007

Location(s)

Schlieren, Lausanne

Legal Form

Strategy Board

Foundation

Dr. Pascale Vonmont

Dr. Suzanne Schenk

Dr. Igor Fisch

\section{Contact}

Contact Person

Philip Hassler, Co-Managing Director

Address

Venture Kick c/o Venturelab, startup space

Wiesenstrasse 5 8952 Schlieren

E-mail info@venturekick.ch

Website www.venturekick.ch 